\title{
Partitioning of pulmonary vascular resistances in group 2 pulmonary hypertension: insightful suggestions for bridging haemodynamics to underlying mechanisms
}

\author{
Marco Guazzi $i^{1,2}$ \\ Affiliations: ${ }^{1}$ Dept of Biomedical Sciences for Health, University of Milan, IRCCS Policlinico San Donato, \\ Milan, Italy. ${ }^{2}$ Dept of Cardiology University, IRCCS Policlinico San Donato, Milan, Italy.
}

Correspondence: Marco Guazzi, University of Milano, Dept of Biomedical Sciences for Health, Heart Failure Unit-Cardiology, IRCCS Policlinico San Donato, Piazza E. Malan 2, 20097 San Donato Milanese, Milano, Italy. E-mail: marco.guazzidunimi.it

@ERSpublications

The relevance of partitioning pulmonary vascular resistance in the different determinants in group 2 PH represent a step forward in the diagnosis and definition of left-sided PH http://ow.ly/gx6330jTzwu

Cite this article as: Guazzi M. Partitioning of pulmonary vascular resistances in group 2 pulmonary hypertension: insightful suggestions for bridging haemodynamics to underlying mechanisms. Eur Respir $J$ 2018; 51: 1800816 [https://doi.org/10.1183/13993003.00816-2018].

Haemodynamic profiling of group 2 pulmonary hypertension $(\mathrm{PH})$ is constantly evolving, and poses a number of challenges in terms of diagnosis and staging [1]. Given that the haemodynamic signature of left-sided $\mathrm{PH}$ is the increased pulmonary arterial wedge pressure (PAWP) at rest $(>15 \mathrm{mmHg})$ with a mean pulmonary arterial pressure $(\mathrm{mPAP}) \geqslant 25 \mathrm{mmHg}$, some diagnostic uncertainty may occur when mPAP is elevated in the presence of a non-dilated left ventricular (LV) chamber with preserved LV ejection fraction and a PAWP at rest within the upper normal limit (range 12-15 $\mathrm{mmHg}$ ) [2]. Mounting evidence suggests that a haemodynamic assessment under physical stress [3] or fluid load [4, 5] may be of help to distinguish the primary from secondary origin of $\mathrm{PH}$.

More importantly, there is still open debate on the optimal haemodynamic definition applied to the different stages. The controversy stands on the best measure that should be sensitive enough to differentiate between isolated backward transmission of pressure from the left atrium to the pulmonary vascular system, i.e. combined passive post-capillary pulmonary hypertension (Ipc- $\mathrm{PH}$ ) and the development of a vascular disease process that adds on to post-capillary $\mathrm{PH}$, i.e. pre- and post-capillary pulmonary hypertension (Cpc-PH) [1]. Indeed, haemodynamic definitions may not comply with intrinsic biological changes in the pulmonary small vessels intended not only as small arteries and capillaries but also veins [6].

Although it is axiomatic that in either group 1 or $2 \mathrm{PH}$, assessment of pulmonary vascular resistance (PVR) plays a key role in signalling a pre-capillary component [7], there has been discussion for a while as to whether incorporation of the diastolic pressure gradient (DPG) (diastolic pulmonary pressure minus $\mathrm{PAWP}) \geqslant 7 \mathrm{mmHg}$ may further refine the $\mathrm{Cpc}-\mathrm{PH}$ condition [8-11]. In 2013, the document drawn up at the Nice conference prioritised a $\mathrm{DPG} \geqslant 7 \mathrm{mmHg}$ as the reference variable for a Cpc-PH diagnosis [12]. However, the most recent evidence has clarified that the use of DPG may not be functional to the question 
because the proportion of subjects with a DPG $\geqslant 7 \mathrm{mmHg}$ and a PVR $\leqslant 3$ Wood units (WU) is trivial compared to the larger amount of patients with a PVR $>3 \mathrm{WU}$ and a DPG $<7 \mathrm{mmHg}$ [7]. Based also on the predominant evidence that DPG $\geqslant 7 \mathrm{mmHg}$ was not prognostic in most subsequent case series, in the latest European Society of Cardiology/European Respiratory Society guidelines, PVR was reintroduced as a pivotal variable to detect the transition to a pulmonary vascular disease, and $\mathrm{Cpc}-\mathrm{PH}$ was defined as a $\mathrm{mPAP} \geqslant 25 \mathrm{mmHg}$, DPG $\geqslant 7 \mathrm{mmHg}$ and/or PVR $>3 \mathrm{WU}$ [13].

These matters of discussion remain significant because they affect a thorough diagnosis and the reliability of haemodynamic phenotyping of $\mathrm{PH}$ through different stages, with remarkable implications on the appropriate selection for interventional strategies out of pulmonary pressure control.

In group $2 \mathrm{PH}$, there is now wide evidence that a PVR $>3 \mathrm{WU}$ occurs in the most advanced stages of the disease with the transition to a "vascular phenotype" [14]; it is highly prognostic [7, 15] and remains a reference variable for pharmacological test reversibility in heart transplant candidates [16]. Although PVR gives a measure of resistance to the blood flow through the lungs, it does not account for the contribution of pulsatile loading, account for the fact that pulmonary artery pressure is higher than atrial pressure at zero flow [17]. "Pulsatile loading" is interpreted as an increase in right ventricular (RV) afterload that primarily depends on LV filling pressure and left atrial (LA) function. Under physiological conditions, this accounts for the $50 \%$ of total load on the right ventricle. In heart failure (HF), there are haemodynamic and circumstantial factors that account for a larger increase in pulsatile load due to LA stiffening, occurrence of atrial fibrillation and mitral incompetence [14]. PAWP is a major descriptor of an increased pulsatile loading but it is uncertain how sensitive PAWP may be to capture the increase in volume load rather than pressure. Moreover, partitioning PVR in the contribution of each component of pulmonary vasculature toward total resistance seems relevant to clarify those aspects and, ultimately, it has the potential to focus on interventions that might be effective in modulating the afterload in the specific portions of the pulmonary circulation [18-20].

In this issue of the European Respiratory Journal, GERGES et al. [21] report the results of partitioning PVR into its components in a group of patients with left-sided $\mathrm{PH}$ compared to idiopathic pulmonary hypertension (iPAH). Cardiac patients were grouped as $\mathrm{Ipc}-\mathrm{PH}$ and $\mathrm{Cpc}-\mathrm{PH}$, and the $\mathrm{Cpc}-\mathrm{PH}$ phenotype was defined according to the Nice Conference definition, i.e. a DPG $\geqslant 7 \mathrm{mmHg}$ and a PVR $>3 \mathrm{WU}$, based on a reappraisal made by the authors themselves [22].

Of the different techniques proposed for partitioning PVR, they chose the pulmonary artery wave occlusion method [19]. This approach allows for an estimate of the effective capillary pressure $\left(P_{c^{\prime}}\right)$ using a bi-exponential fitting of the pressure decay curve between the moment of occlusion and mPAWP with normalisation to mPAP. Thus, using $P_{c^{\prime}}$, PVR was partitioned into larger arterial upstream resistance ( $R$ up) and downstream resistance $(R \mathrm{ds})$ due to small arterial plus venous components. Contribution of PVR partitioning was addressed also during acute vasoreactivity by inhaled nitric oxide (iNO) testing.

Major findings are that RV afterload was significantly and similarly higher in iPAH and Cpc-PH. In Ipc- $\mathrm{PH}, R$ up and the gradient between $P_{\mathrm{c}^{\prime}}$ and PAWP were respectively higher and smaller than in $\mathrm{Cpc}-\mathrm{PH}$ and iPAH. Rup was significantly and negatively correlated with DPG along the left-heart disease PH stages.

iNO induced a decrease in $P_{c^{\prime}}$ in $\mathrm{Cpc}-\mathrm{PH}$ and a significant increase in $P_{c^{\prime}}$ was observed in Ipc-PH. In addition, iNO induced a significant increase in $\mathrm{Rup}$ in $\mathrm{Cpc}-\mathrm{PH}$ and no changes in Ipc-PH. The pulmonary haemodynamic response to vasoreactive challenge was variable according to classical responders and nonresponders. Overall, iNO induced a significant improvement in cardiac output, mPAP, PVR, DPG and pulmonary arterial compliance (PAC) in $\mathrm{Cpc}-\mathrm{PH}$ and $\mathrm{iPAH}$. In Ipc- $\mathrm{PH}$, only an increase in PAWP was observed.

The article is valuable because for the first time, it focusses interest on the frequently overlooked and understudied role of pulsatile versus resistive loading in the progression of left-sided PH. The findings prompt us to question how much the definition of "passive" $\mathrm{PH}$ is appropriate, and indirectly lend credit to the observations that there are patients with still a PVR $<3 \mathrm{WU}$ and DPG within normal limits who already exhibit some degrees of RV to pulmonary circulation uncoupling, likely as a consequence of the early right heart inability to adapt to an increasing $R$ up despite a preserved $R \mathrm{ds}$ [23]. Application of PVR partitioning is attractive for discerning $\mathrm{PH}$ of cardiac from that of noncardiac origin in case of the so-called "atypical iPAH", a condition characterised by a mPAP $\geqslant 25 \mathrm{mmHg}$, a PAWP within the upper limit of normal and more than three associated cardiovascular risk factors, primarily obesity [2]. Defining the relative contribution of $R$ up and $R$ ds in this group of patients appears to be a more straightforward haemodynamic diagnostic procedure than fluid loading challenge, the haemodynamic response and cut-off of which are quite unpredictable, especially considering that in HF most of the fluid infused can easily transit in the interstitium [24]. 
In general, subjects were optimally treated according to guidelines, suggesting that therapies, excluding diuretics or neuro-hormonal blocker agents, that may modulate and reverse the abnormal pulsatile loading component due to a loss of LA compliance and pulmonary veins remodelling, represent an unmet need.

Unfortunately, patients with significant mitral regurgitation and atrial fibrillation who represent the majority of left-sided $\mathrm{PH}$ populations with $\mathrm{Cpc}-\mathrm{PH}$ were excluded, preventing speculation on the respective contribution of these determinants on $R$ up. These aspects definitively arise as important drawbacks that add to the lack of data reproducibility, primarily for DPG measures, the reliability of which is dependent on small factors [25], and poor subgroup characterisation, especially in response to iNO challenge due to the limited number of patients for each group. The study seems also underpowered for drawing solid conclusions on the significance of other haemodynamic measures such as PAC in the context of PVR partitioning and in response to iNO. The correlation between DPG and Rup cannot be taken as supportive of a Cpc-PH definition based on DPG $\geqslant 7 \mathrm{mmHg}$, as suggested by the authors, because the correlation is weak and most points are outside the $95 \%$ confidence interval. Furthermore, the current definition of Cpc-PH actually prioritises PVR and not DPG.

It is intriguing to consider that the effectiveness of specific interventions, such as the use of phosphodiesterase-5 inhibition, that have shown to benefit some subsets of group $2 \mathrm{PH}$ [26-28] should be further confirmed or dismissed by looking at the PVR partitioning before and after treatment.

GERGes et al. [21] have the undisputed merit of having enriched the haemodynamic definition of group 2 $\mathrm{PH}$ by looking at afterload in its different components, providing a direct demonstration of how and to what extent fixed versus pulsatile loading may contribute to $\mathrm{PH}$ pathogenesis, yielding to a more precise definition and diagnosis. Whereas these haemodynamic derivations seem now feasible only in experienced laboratories, and further validation and consensus on how they may predict the response to interventions is still missing, we can indeed appreciate the insightful messages and the steps forward in a field of high expectations, i.e. bridging haemodynamics to underlying mechanisms in the complex process of phenotyping group $2 \mathrm{PH}$.

Conflict of interest: None declared.

Support statement: M. Guazzi is supported by a grant from the Monzino Foundation (Milan, Italy).

\section{References}

1 Guazzi M, Naeije R. Pulmonary hypertension in heart failure: pathophysiology, pathobiology, and emerging clinical perspectives. J Am Coll Cardiol 2017; 69: 1718-1734.

2 Opitz CF, Hoeper MM, Gibbs JS, et al. Pre-capillary, combined, and post-capillary pulmonary hypertension: a pathophysiological continuum. J Am Coll Cardiol 2016; 68: 368-378.

3 Maor E, Grossman Y, Balmor RG, et al. Exercise haemodynamics may unmask the diagnosis of diastolic dysfunction among patients with pulmonary hypertension. Eur J Heart Fail 2015; 17: 151-158.

4 D'Alto $\mathrm{M}$, Romeo E, Argiento $\mathrm{P}$, et al. Clinical relevance of fluid challenge in patients evaluated for pulmonary hypertension. Chest 2017; 151: 119-126.

5 Andersen MJ, Olson TP, Melenovsky V, et al. Differential hemodynamic effects of exercise and volume expansion in people with and without heart failure. Circ Heart Fail 2015; 8: 41-48.

6 Fayyaz AU, Edwards WD, Maleszewski JJ, et al. Global pulmonary vascular remodeling in pulmonary hypertension associated with heart failure and preserved or reduced ejection fraction. Circulation 2018; 137: 1796-1810.

7 Palazzini M, Dardi F, Manes A, et al. Pulmonary hypertension due to left heart disease: analysis of survival according to the haemodynamic classification of the 2015 ESC/ERS guidelines and insights for future changes. Eur J Heart Fail 2018; 20: 248-255.

8 Gerges C, Gerges M, Skoro-Sajer N, et al. Hemodynamic thresholds for precapillary pulmonary hypertension. Chest 2016; 149: 1061-1073.

9 Gerges M, Gerges C, Pistritto AM, et al. Pulmonary hypertension in heart failure. Epidemiology, right ventricular function, and survival. Am J Respir Crit Care Med 2015; 192: 1234-1246.

10 Tedford RJ, Beaty CA, Mathai SC, et al. Prognostic value of the pre-transplant diastolic pulmonary artery pressure-to-pulmonary capillary wedge pressure gradient in cardiac transplant recipients with pulmonary hypertension. J Heart Lung Transplant 2014; 33: 289-297.

11 Tampakakis E, Shah SJ, Borlaug BA, et al. Pulmonary effective arterial elastance as a measure of right ventricular afterload and its prognostic value in pulmonary hypertension due to left heart disease. Circ Heart Fail 2018; 11: e004436.

12 Vachiery JL, Adir Y, Barbera JA, et al. Pulmonary hypertension due to left heart diseases. J Am Coll Cardiol 2013; 62: D100-D108.

13 Galie N, Humbert M, Vachiery JL, et al. 2015 ESC/ERS Guidelines for the diagnosis and treatment of pulmonary hypertension: The Joint Task Force for the Diagnosis and Treatment of Pulmonary Hypertension of the European Society of Cardiology (ESC) and the European Respiratory Society (ERS): Endorsed by: Association for European Paediatric and Congenital Cardiology (AEPC), International Society for Heart and Lung Transplantation (ISHLT). Eur Heart J 2016; 37: 67-119.

14 Guazzi M, Borlaug BA. Pulmonary hypertension due to left heart disease. Circulation 2012; 126: 975-990. 
15 Miller WL, Grill DE, Borlaug BA. Clinical features, hemodynamics, and outcomes of pulmonary hypertension due to chronic heart failure with reduced ejection fraction: pulmonary hypertension and heart failure. JACC Heart Fail 2013; 1: 290-299.

16 Mehra MR, Canter CE, Hannan MM, et al. The 2016 International Society for Heart Lung Transplantation listing criteria for heart transplantation: a 10-year update. J Heart Lung Transplant 2016; 35: 1-23.

17 Permutt S, Riley RL. Hemodynamics of collapsible vessels with tone: the vascular waterfall. J Appl Physiol 1963; 18: 924-932.

18 Hakim TS, Michel RP, Chang HK. Partitioning of pulmonary vascular resistance in dogs by arterial and venous occlusion. J Appl Physiol Respir Environ Exerc Physiol 1982; 52: 710-715.

19 Kafi SA, Melot C, Vachiery JL, et al. Partitioning of pulmonary vascular resistance in primary pulmonary hypertension. J Am Coll Cardiol 1998; 31: 1372-1376.

20 Kim NH, Fesler P, Channick RN, et al. Preoperative partitioning of pulmonary vascular resistance correlates with early outcome after thromboendarterectomy for chronic thromboembolic pulmonary hypertension. Circulation 2004; 109: 18-22.

21 Gerges C, Gerges M, Fesler P, et al. In-depth haemodynamic phenotyping of pulmonary hypertension due to left heart disease. Eur Respir J 2018; 51: 1800067.

22 Gerges M, Gerges C, Lang IM. How to define pulmonary hypertension due to left heart disease. Eur Respir J 2016; 48: 553-555.

23 Guazzi M, Dixon D, Labate V, et al. RV contractile function and its coupling to pulmonary circulation in heart failure with preserved ejection fraction: stratification of clinical phenotypes and outcomes. JACC Cardiovasc Imaging 2017; 10: 1211-1221.

24 Guazzi M. Letter regarding article, "Differential hemodynamic effects of exercise and volume expansion in people with and without heart failure". Circulation 2015; 132: e160.

25 Naeije R. Measurement to predict survival: the case of diastolic pulmonary gradient. JACC Heart Fail 2015; 3: 425.

26 Lewis GD, Lachmann J, Camuso J, et al. Sildenafil improves exercise hemodynamics and oxygen uptake in patients with systolic heart failure. Circulation 2007; 115: 59-66.

27 Malhotra R, Dhakal BP, Eisman AS, et al. Pulmonary vascular distensibility predicts pulmonary hypertension severity, exercise capacity, and survival in heart failure. Circ Heart Fail 2016; 9: e003011.

28 Guazzi M, Vicenzi M, Arena R, et al. Pulmonary hypertension in heart failure with preserved ejection fraction: a target of phosphodiesterase-5 inhibition in a 1-year study. Circulation 2011; 124: 164-174. 